\section{Amygdalectomy and mixed reinforcement schedule contrast effects}

\author{
PETER G. HENKE \\ Muskingum College, New Concord, Ohio 43762
}

Four controls and four amygdalectomized rats were trained to leverpress on a mixed schedule of reinforcement in which $5 \mathrm{~min}$ of variable-interval reinforcement alternated with $5 \mathrm{~min}$ of extinction. Control animals increased responding in the variable-interval component over baseline rates (i.e., behavioral contrast), whereas amygdalectomized animals did not. No significant differences between groups were found during extinction. The lack of contrast effects in amygdaloid animals was attributed to a reduction in extinction-induced frustration.

Behavioral contrast may be defined as opposing response changes in multicomponent reinforcement schedules. Typically, both components of the reinforcement schedule are correlated with different exteroceptive stimuli (multiple schedule) and, following stable responding, the reinforcement conditions in one component are changed, whereas the second component remains unaltered For example, Reynolds (1961) found that switching one component of a multiple variable-interval schedule to extinction produced response rate increases in the unchanged component (i.e., behavioral contrast). Recently, contrast effects have been reported in mixed reinforcement schedules in which no explicit discriminative stimuli were correlated with the schedule components (Pear \& Wilkie, 1970).

The present study sought to determine if amygdalectomy would prevent the occurrence of behavioral contrast in a mixed schedule of reinforcement. Henke and Maxwell ${ }^{1}$ found that lesions in the amygdaloid complex eliminated the frustration effect (FE) that is commonly observed in double runway studies. FE refers to an invigoration of responding in the second alley following nonreinforcement in the first goalbox (e.g., Amsel \& Roussel, 1952). However, the effect has also been reported in operant studies (Davenpoit, Flaherty, \& Dryud, 1966), and it has been argued that behavioral contrast and FE may have similar immediate causes (Scull, Davis, \& Amsel, 1970).

\section{SUBJECTS}

Eight experimentally naive male Wistar rats were used. They were housed in individual cages. At the beginning of the study, their ages ranged from 120 to 140 days.

$$
\text { APPARATUS }
$$

Training occurred in Lehigh Valley operant chambers in which only the right-hand lever was present. Reinforcement was provided by a 0.2-ml water dipper. The apparatus was enclosed in a sound-attenuating cubicle.

\section{SURGERY AND HISTOLOGY}

Prior to any behavioral training, four animals were given bilateral RF (Grass LM-4) lesions in the amygdala through stereotaxically guided stainless steel electrodes. Surgical procedures were identical to those discribed previously (Henke, Allen, \& Davison, 1972). Two animals received control operations that were similar to lesion placements, except that the electrode was not lowered into the brain. At the end of the study, the animals were perfused with saline and $10 \%$ Formalin. After dehydration and embedding in celloidin, sections were cut at 30 microns. Every fifth section was mounted and stained with cresyl violet.

\section{PROCEDURE}

Following a 2-week recovery period, all Ss were placed on a $22-\mathrm{h}$ reinforcement. water-deprivation schedule. This schedule was in effect for 7 days before training began and was maintained throughout the study. Food was always available in the home cages, and water was given after each 1-h training session for an additional 1-h period.

During magazine training, the dipper automatically delivered free reinforcements in $1-\mathrm{min}$ intervals until 60 reinforcements had been given. The next day, all animals were manually shaped to press the lever, and the session terminated after 30-40 leverpresses had been continuously reinforced.

After three daily 1 -h sessions on a variable-interval (VI) 10 -sec schedule of reinforcement, Ss were shifted to a VI 20-sec schedule for 15 sessions. After VI 20-sec training, the Ss were placed on a mixed VI 20-extinction reinforcement schedule for 25 sessions. The VI and extinction components alternated with each other every $5 \mathrm{~min}$.

\section{RESULTS}

Lesions typically extended from the crossings of the anterior commissure approximately $2.00 \mathrm{~mm}$ posteriorly. The lateral, basal lateral, basal medial, central, and cortical nuclei were destroyed in all four animals; however, the medial nucleus was only slightly damaged. Damage to pyriform cortex and stria terminalis was found in each S.

The behavioral data are represented in Fig. 1. It shows that control animals responded at increasing rates during the VI component of the mixed reinforcement schedule: On the other

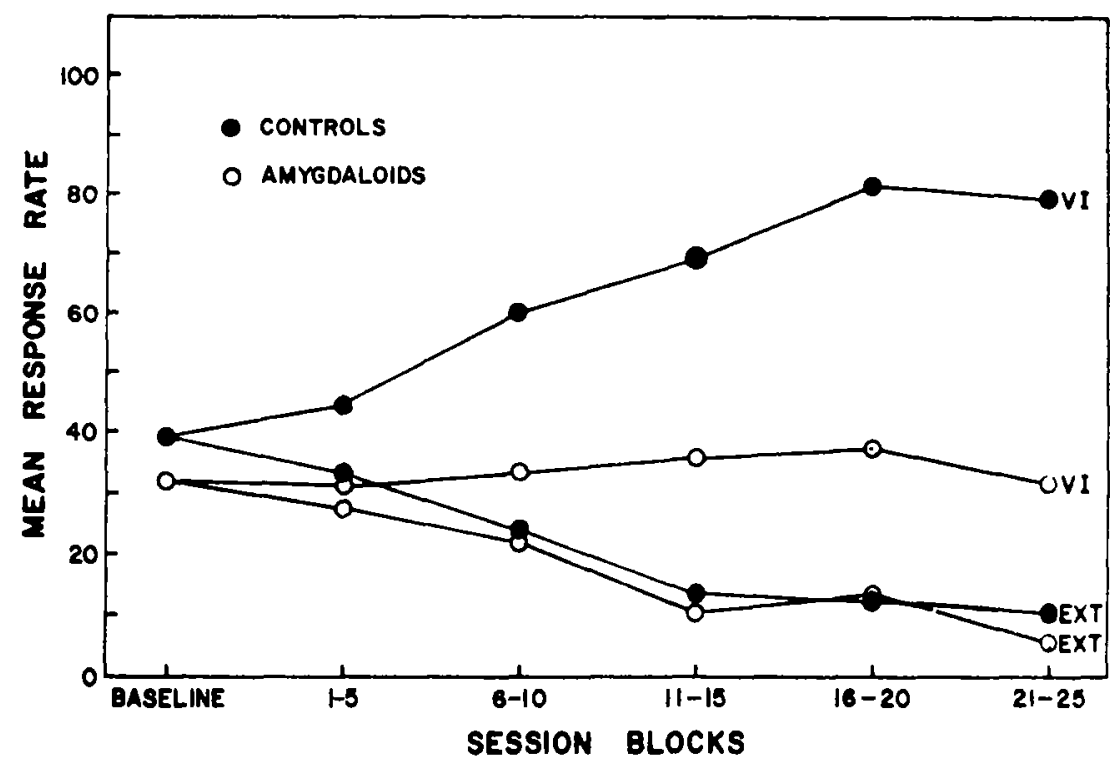

Fig. 1. Mean responses/minute of control and amygdaloid animals averaged over consecutive five-session blocks on a mixed VI 20-extinction schedule of 
hand, amygdalectomized Ss did not show behavioral contrast effects. The Friedman test by ranks (Siegel, 1956) confirmed that the increase in response rates relative to baseline rates was reliable for the control group $\left(x^{2}\right.$ ranks $=19.01, \mathrm{df}=5, \mathrm{p}<.01$ ), whereas no significant changes were found for the amygdaloid Ss $(p>.10)$. Figure 1 also indicates that both groups reached similar baseline performance before the extinction component was introduced. Mean session baseline rates (averaged over five sessions) were $38.7, \mathrm{SD}=3.5$, and $34.1, \mathrm{SD}=4.1$, for control and am y gdalectomized groups, respectively.

Response rates during the extinction component decreased in a similar manner for both groups. The Friedman test indicated that the decline was reliable for the controls $\left(\chi^{2}\right.$ ranks $\left.=18.30, \mathrm{df}=5, \mathrm{p}<.01\right)$ and the amygdaloid Ss $\left(x^{2}\right.$ ranks $=18.10$, $\mathrm{df}=5, \mathrm{p}<.01)$. There was no significant difference between groups at any point during extinction (Mann-Whitney $U$ test, $p>.10$ ). DISCUSSION

The data of control Ss essentially replicated the results of an earlier study (Pear \& Wilkie, 1970). The response rate decrease during extinction was accompanied by an increase in rate in the reinforced component of the mixed reinforcement schedule (i.e., behavioral contrast). However, amygdalectomy prevented the occurrence of contrast effects. Similar results have been found in multiple reinforcement schedule responding (Henke et al, 1972) and after several daily extinction sessions were interpolated between successive reinforcement periods (Henke \& Bunnell, 1971).

One explanation of behavioral contrast assumes that the increase in rate of responding represents by-products of frustration elicited by nonreinforcement during the extinction component (Scull et al, 1970). Relevant to this interpretation are data that indicate that lesions in the amygdala altered the emotionality of animals. Several studies have shown that amygdalectomy produced placidity in domesticated as well as wild animals (see Goddard, 1964). Thus, it may be concluded that the lesion impairment found in the present study may be related to deficiencies in forming affective responses to changed reinforcement conditions. The fact that amygdaloid lesions virtually eliminated the frustration effect in the double runway (Henke \& Maxwell) supports this conclusion.

\section{REFERENCES}

AMSEL, A., \& ROUSSEL, J. Motivational properties of frustration: i. Effect on a running response of the addition of frustration to the motivational complex. Journal of Experimental Psychology, $1952,43,363-368$.

DAVENPORT, J. W., FLAHERTY, C. F., \& DRYUD, J. P. Temporal persistence of frustration effects in monkeys and rats. Psychonomic Science, 1966, 6, 411-412. GODDARD, G. V. Functions of the amygdala. Psychological Bulletin, 1964, 62, 89-109.

HENKE, P. G., ALLEN, J. D., \& DAVISON C. Effect of lesions in the amygdala on behavioral contrast. Physiology \& Behavior, 1972, 8, 173-176.

HENKE, P. G.. \& BUNNEEL, B. N Reinforcement and extinction interactions after limbic lesions in rats. Communications in Behavioral Biology, 1971, 6, 329-333.

PEAR, J. J., \& WILKIE, D. M. Behavioral contrast in mixed schedules of reinforcement. Psychonomic Science, $1970,20,167-168$.

REYNOLDS, G. S. Behavioral contrast. Journal of the Experimental Analysis of Behavior, 1961, 4, 57-71.

SCULL, J., DAVIS, K., \& AMSEL, A Behavioral contrast and frustration effect in multiple and mixed fixed-interval schedules in the rat. Journal of Comparative \& Physiological Psychology, $1970,71,478-483$.

SIEGEL, S. Nonparametric statistics for the behavioral sciences. New York: McGraw-Hill, 1956.

NOTE

1. Henke, P, G., \& Maxwell, D. Amygdalectomy and the frustration effect, in preparation. 